\title{
Aprendizaje, memoria y comprensión de textos expositivos. Dos estudios de intervención sobre el texto
}

\author{
JUAN ANTONIO GARCIA-MADRUGA \\ JUAN LUQUE \\ JESUS MARTIN \\ Universidad de Madrid
}

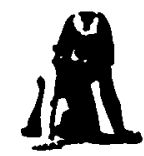

Resumen

Este artículo se encuadra en el área de investigación de la psicología de la comprensión de textos. Se realiza una breve revisión de conceptos fundamentales en orden a una mejor comprensión de los dos estudios que a continuación se presentan. La manipulación experimental se centra sobre el texto. El principal objetivo de ambos estudios consiste en comprobar la eficacia diferencial de dos tipos de ayuda -esquema y objetivos- sobre los procesos de memoria, comprensión y recuperación de la información procedente de textos expositivos. Un segundo objetivo es la comprobación del efecto de algunas modificaciones experimentales en los textos utilizados. La pauta de resultados obtenidos es consistente con la idea de que la presentación de un esquema previo a la lectura del texto optimiza los procesos de almacenamiento y recuperación de la información. Mostrándose, sin embargo, los objetivos como una ayuda de efectos muy limitados y especificos, o incluso nulos.

Palabras clave: Comprensión de textos, memoria de prosa, variables de texto, tipos de ayuda, objetivos, esquemas.

\section{Learning, memory and comprehension of expository texts: two texts intervention studies}

\section{Abstract}

The theoretical context of this paper is that of text comprebension psycbology. We present a brief review of some basic concepts that we bope will help to gain a better understanding of the two studies later introduced. The experimental intervention is centred on the text and not on the subjects. The main goal of both studies is to compare the differential eficacy of two types of aids-outlines and objectives- with regard to memory processes, comprebension and information retrieval from expository texts. A second goal is to test the effect of some experimental modifications on texts. The observed pattern of results supports the idea that storage and information retrieval processes are optimized by the introduction of an outline before the reading of the text. On the other band, objectives seem to be a very limited and specific aid.

Key words: Text comprebension, memory for prose, text variables, types of aid, learning objectives, outline.

Agradecimientos: Estos dos estudios han sido realizados gracias a dos ayudas concedidas por el CIDE, organismo al que agradecemos su apoyo. Asimismo queremos agradecer su colaboración á Carlos Santamaría Moreno y a los restantes miembros del equipo investigador.

Dirección del autor: Universidad Nacional de Educación a Distancia. Departamento de Psicología de la Personalidad Social y Evolutiva (P. Box). Apartado 50.487. Madrid.

Original recibido: mayo 1989. Revisión recibida: junio 1989. Aceptado: julio 1989. 
Este artículo pretende evaluar, mediante resultados experimentales, la eficacia de dos tipos de ayuda -esquema y objetivos- para optimizar los procesos de comprensión y recuerdo de textos expositivos. En este sentido queremos destacar que los estudios que a continuación se presentan constituyen el inicio de una línea de investigación y poseen un carácter fundamentalmente exploratorio. No obstante, en el segundo de ellos, se intenta replicar y profundizar en algunos resultados concretos del primer estudio. Antes de la presentación de los trabajos experimentales y con la intención de facilitar su inclusión en el marco teórico coherente, describiremos, sucintamente, algunas ideas que han sido fundamentales en la actual articulación de la psicología de la comprensión de textos.

El interés de la psicología educativa en la mejora del aprendizaje de textos escritos había encontrado en el ateoricismo una de las principales dificultades para su desarrollo. En los últimos veinte años han ido apareciendo una serie de modelos sobre la organización de textos escritos pertenecientes a dos tradiciones. Autores como Ausubel (1968; Novak, 1977), desde una concepción teórica cognoscitiva han mostrado cómo se articulan los nuevos contenidos en las estructuras cognoscitivas mediante la presentación de organizadores previos. Rothkopf (1970; Rothkopf y Kaplan, 1972) dentro de la tradición conductista fundamentó sus estudios en las conductas matemagénicas (generadoras de habilidades de aprendizaje) y el efecto que la utilización de objetivos y preguntas tiene sobre el aprendizaje de un texto escrito. Por su parte, Gagné (1977) centraba su modelo en las «habilidades intelectuales» y en las jerarquías y resultados del aprendizaje.

Algunos de estos modelos tendían ya a asumir claramente que el sujeto del aprendizaje es un receptor activo de información que integra los nuevos conocimientos en estructuras cognoscitivas previas, alejándose de un modelo pasivo de aprendizaje humano. Sin embargo, ha sido una tradición más reciente, centrada más en la memoria que en el aprendizaje, la que está dotando de un contexto teórico general a las investigaciones en este campo, salvando, de este modo, las dificultades fundamentales del ateoricismo y resolviendo algunos de los problemas importantes sobre la estructura y organización de estos escritos.

En los últimos años la comprensión de textos ha sido objeto de una atención privilegiada por parte de los investigadores. Los estudios sobre memoria para prosa han mostrado que la comprensión no depende únicamente del texto o de las estructuras cognoscitivas previas del sujeto, sino de una interacción entre el texto, con sus características estructurales, y los esquemas usados por el sujeto (Rumelhart, 1975; Schank y Abelson, 1977; Thorndyke y Hayes-Roth, 1979). Por otro lado, debe destacarse que también los procesos psicológicos básicos que subyacen a la comprensión actúan de forma interactiva y coordinada. El reconocimiento de palabras, el análisis sintáctico y el análisis semántico -una vez automatizados por la práctica- actúan en forma paralela, activándose tan pronto como accede a ellos alguna información. El resultado final de este proceso será una representación mental del discurso o texto que incluirá, además del conjunto de proposiciones que componen un texto, la construcción de un modelo referencial o situacional del significado del mismo (Just y Carpenter, 1987; van Dijk y Kintsh, 1983; Johnson-Laird, 1983). 


\section{La representación de la estructura del texto}

Uno de los principales objetivos de la investigación sobre la comprensión de textos ha sido caracterizar la construcción y representación de la estructura del texto. El hecho experimental básico a este respecto consiste en que la comprensión y el recuerdo de los contenidos de un texto dependen del nivel que ocupen dentro de una estructura jerárquica en la que se constituye la representación mental de un texto. Según este fenómeno llamado "efecto de los niveles» los contenidos e ideas importantes, que ocupan una posición más alta en la jerarquía, serán más probablemente recordados que aquellos que ocupan una posición más baja.

Durante los años setenta se desarrollaron una serie de modelos que trataron de describir y explicar cómo se construía la representación del texto, de forma que respondiese a este efecto de los niveles. El modelo más conocido y completo de esta época es el de Kintsh y van Dijk (1978) del que revisaremos brevemente cómo explica este efecto de los niveles.

Según estos autores, la representación del contenido semántico de un texto que construye el lector, consiste en un conjunto jerarquizado de proposiciones formadas por un predicado y uno o más argumentos. Este conjunto de proposiciones, conectadas entre sí mediante la repetición de argumentos, constituye la microestructura o base del texto. A partir de la base del texto el sujeto construye la macroestructura o representación semántica global del significado del mismo, tal como se manifiesta en los protocolos de recuerdo. Este proceso requiere la aplicación de operadores a la microestructura del texto. Tales operadores se conocen con el nombre de macrorreglas y son tres: supresión, generalización y construcción. Mediante su aplicación se reduce el número de proposiciones (supresión) y se organiza la información manteniendo proposiciones relevantes e incorporando nuevas proposiciones (generalización y construcción). Sin embargo las macrorreglas no garantizan la comprensión del discurso; por sí solas podrían producir abstracciones y generalizaciones sin sentido. Las macrorreglas se aplican bajo el control de las superestructuras esquemáticas que representan los conocimientos previos y propósitos del sujeto y permiten reconocer la estructura de una narración o exposición y aplicar estrategias estructurales (Meyer, 1984) en la recuperación y el recuerdo.

El modelo de Kintsh y van Dijk incluye una descripción del proceso de construcción de la representación del texto. El primer problema que se plantea es cómo se forma una base del texto que sea coherente, para lo cual estos autores proponen tres hipótesis:

1. En primer lugar se comprueba la coherencia referencial de la base del texto. En el caso de que exista solapamiento, o sea, repetición de argumentos entre todas las proposiciones se acepta para el procesamiento posterior. De no ser así se pondrian en marcha los procesos de construcción de inferencias con objeto de conseguir la coherencia.

2. Debido a limitaciones de la memoria en funcionamiento, el procesamiento del texto se realiza secuencialmente, en ciclos, mediante el agrupamiento de varias proposiciones.

3. Para conectar los diferentes ciclos, algunas proposiciones se mantienen, de un ciclo a otro, en un retén (buffer) de la memoria en funcionamiento.

El modelo sostiene que la probabilidad con la que una proposición será almacenada y después reproducida, depende del número de veces que haya sido 
seleccionada o procesada entre un ciclo y otro. La selección de las proposiciones se realiza mediante dos estrategias principalmente: las proposiciones conectadas con otras muchas proposiciones, en otras palabras, las de más alto nivel en la jerarquía, serán seleccionadas preferentemente; la segunda estrategia se basa en la «recencia»; es decir, se seleccionan aquellas proposiciones procesadas más recientemente.

Las deficiencias del modelo de Kintsh y van Dijk (1978), se ponen de manifiesto en su explicación en términos de frecuencia del efecto de los niveles. A esta explicación Meyer (1984) añade otras dos posibilidades: codificación selectiva y recuperación diferencial. La codificación selectiva o hipótesis de la selección se ha visto reforzada recientemente por el hallazgo de diferentes tiempos de lectura para proposiciones de alto y bajo nivel (Cirilo y Foss, 1980): incluyendo una pequeña oración dentro de dos narraciones diferentes de forma que en una ocupaba un lugar alto en la jerarquía y en la otra bajo.

El nuevo modelo de van Dijk y Kintsh (1983), sostiene que no sólo puede variar la cantidad, la frecuencia del procesamiento, sino también la calidad; existiendo proposiciones más importantes por su capacidad para obtener la macroestructura, o por el hecho de formar ellas mismas parte de la macroestructura, y que el lector puede reconocer en el texto y lee más cuidadosamente. Del mismo modo en el nuevo modelo se incluye el componente de recuperación; con este componente se daría cuenta de que el efecto de los niveles no haya sido encontrado en tareas de reconocimiento. El recuerdo estaría en función tanto del almacenamiento como de la recuperación. Además se otorga una mayor importancia a la macroestructura y los esquemas superestructurales que guían tanto la codificación como la recuperación. Por último, el nuevo modelo que van Dijk (1983) llama estratégico, acentúa también el papel de los conocimientos previos y las estrategias de los sujetos en la comprensión y memoria de textos.

\section{La aportación del sujeto}

La creciente importancia otorgada a los procesos constructivos en la comprensión y memoria de textos ha ido unida al desarrollo de las teorías del esquema y a la consiguiente crítica de los modelos de procesamiento de secuencia lineal. Las teorías del esquema conciben la comprensión de textos como un proceso de comprobación de hipótesis, en el que el sujeto, a partir de los indicios que proporciona el texto, genera determinados esquemas-hipótesis que se evalúan contrastándolos con las oraciones sucesivas hasta lograr una interpretación consistente. El peso de las contribuciones cognitivas del sujeto fue puesto de manifiesto por las investigaciones de Bransford y colaboradores (Bransford, Barclay y Franks, 1972; Bransford y Johnson, 1972, 1973; Bransford y McCarrell, 1975) y se vio reforzado y completado por las aportaciones de los conceptos de esquema (Rumelhart, 1980; Rumelhart y Ortony, 1977), marco (Minsky, 1975) y guión (Schank y Abelson, 1977).

Dos son las aportaciones más importantes que realiza el sujeto durante la lectura de un texto: las inferencias y los conocimientos previos. Si son fundamentales en la articulación teórica de la psicología de la comprensión de textos los conceptos hasta ahora reseñados, no lo es menos, el papel que actualmente se le atribuye a las inferencias en la comprensión del lenguaje en general. Clark (1975) distingue entre inferencias «hacia atrás» que permiten, conectando los 
significados de las diferentes ý sucesivas oraciones, crear una cadena causal de conceptualizaciones que constituyan una representación coherente del texto; e inferencias «hacia adelante» que permiten predecir los hechos o acontecimientos posteriores. Por otra parte los conocimientos que el sujeto aporta pueden ser clasificados en tres grandes clases. En primer lugar, el conocimiento específico del tema o asunto acerca del cual trata el texto en cuestión. En segundo lugar, el conocimiento general del mundo, de las relaciones sociales y de las causas y características que son comunes en muchos campos y situaciones diferentes. En tercer lugar, el conocimiento de las estructuras retóricas superiores que caracterizan y limitan la comunicación escrita, las superestructuras de las que habla van Dijk.

\section{LA INTERVENCION SOBRE EL TEXTO}

Tenemos, por tanto, que el contexto teórico actual donde están desarrollándose las investigaciones en este campo, es el fruto de la convergencia entre la psicología educativa y las aportaciones de la psicología cognitiva. Como anteriormente se citaba, la psicología educativa ha estudiado la repercusión de toda una gama de procedimientos para la mejora del aprendizaje de textos, desde posiciones teóricas muy diversas. Por otro lado, tanto desde la psicolingüística como desde las teorías constructivistas de la memoria de prosa, se ha investigado el efecto de ciertos estímulos e indicios iniciales que favorecen el procesamiento de textos.

Nuestros trabajos han comenzado, del mismo modo, por tomar como objetivo de intervención la manipulación de las variables que afectan al texto. Hemos clasificado y aplicado dos tipos principales de técnicas. Por una parte aquellos procedimientos que se centran en la manipulación de las variables intratextuales y que afectan directamente a la estructura del texto. En este grupo incluimos técnicas como la modificación de la organización o secuencia general del discurso, la simplificación léxica y sintáctica, y el uso de conectivos o fórmulas retóricas. Estas técnicas tienen su principal origen en la psicolinguústica y la psicología cognitiva, habiéndose aplicado en la confección de nuestros textos experimentales un tratamiento común compuesto por varios de estos procedimientos. El segundo gran apartado de clasificación lo compondrían aquellos procedimientos que no alteran el texto pero sobre cuyo recuerdo se espera que ejerzan influencia. Este es el grupo más amplio y en el que se incluyen los organizadores previos, los títulos y sumarios, los objetivos, etc... Dos de estas técnicas son especialmente relevantes para nuestros estudios. La aplicación de objetivos, desarrollada por Rothkopf desde una posición conductista, constituirá una parte de nuestras condiciones experimentales. Por otro lado, cabe resaltar la similitud entre los organizadores previos y nuestro esquema, el cual constituye el otro polo de la contrastación experimental.

Los organizadores previos forman parte de la concepción teórica de Ausubel (1968; Ausubel, Novak y Hanesian, 1978), desarrollada desde una perspectiva típicamente cognoscitiva sobre el aprendizaje significativo. Los organizadores previos son un tipo de ayuda previa caracterizada por el hecho de que su nivel de abstracción y generalidad es superior al del nuevo material que se va a aprender. El esquema es también una ayuda introductoria que actúa menos sobre la superestructura del texto (es decir, a un nivel de abstracción y generali- 
dad superior) y más sobre la propia macroestructura. Ambos utilizan una misma estrategia cognoscitiva de tipo deductivo, que pone el acento en lograr una mejor comprensión global: activar las estructuras conceptuales en las que debe ser integrado el texto.

Uno de los problemas fundamentales en la investigación educativa sobre la memoria de textos es la necesidad de establecer comparaciones cuantitativas. Para ello se han utilizado métodos de fraccionamiento del texto en unidades de menor tamaño aunque la forma óptima de realizar tal descomposición es un problema abierto (Schnotz, 1984). El modelo de análisis proposicional que aplicaremos incluye unidades de orden jerárquico superior a la proposición. El texto original quedó dividido en 67 proposiciones, agrupadas en 11 escenarios y éstos a su vez en 5 bloques. Los escenarios y bloques pretenden captar la comprensión y memoria estructural de estas unidades de orden superior a la proposición en los protocolos de rẹcuerdo de los sujetos, superando lo que podría resultar una medida simple de la capacidad de almacenamiento a largo plazo. De este modo el cómputo de los resultados se orienta a cubrir dos áreas fundamentales de la memoria de textos. En primer lugar, la memoria reproductiva de los datos y conceptos inferiores en la jerarquía o microestructura del texto, cuyo cómputo se obtuvo mediante la comparación de los protocolos con la descomposición o tabla de proposiciones del texto original. En segundo lugar, el cómputo de los conceptos de nivel macroestructural que incluía los escenarios y bloques. Por último, realizamos un cómputo de aquellos aspectos del protocolo que no aparecen en el texto original, que pueden considerarse la parte creativa de la lectura y a las que hemos denominado, en un sentido laxo, inferencias.

\section{PRIMER ESTUDIO}

\section{Objetivos e hipótesis}

El objetivo general fue comprobar la eficacia diferencial de los dos tipos de ayuda utilizados. Este objetivo se concreta en dos hipótesis:

La primera mantiene que las modificaciones introducidas en los textos experimentales deberán producir una mejora en el aprendizaje (postest) y retención (demora) de los textos $\mathrm{B}$ y $\mathrm{C}$, respecto al texto control A.

La segunda de nuestras hipótesis propone al esquema como la ayuda más eficaz. De este modo, la utilización de un esquema previo en el texto experimental B producirá una mejora en los resultados respecto a los otros dos grupos. Esta mejora en los resultados debe manifestarse en las puntuaciones de recuerdo y comprensión, y en las de aprendizaje intencional, en el postest y en la demora.

\section{METODO}

\section{Materiales y diseño}

El texto control A fue extraído de los contenidos de una antigua Unidad Didáctica de Psicología Evolutiva de la UNED. A partir de éste se diseñaron y realizaron dos textos experimentales $(\mathrm{B} \mathrm{y} \mathrm{C}$ ) con el mismo contenido semántico. 
Las modificaciones introducidas en loș textos experimentales fueron de tres tipos. En primer lugar, realizamos algunas modificaciones relativas a la estructura lógica del texto; cambios en la organización del mismo y en la secuencia de los contenidos; utilización en los textos experimentales de marcadores retóricos diferentes; y utilización del espacio para resaltar la estructura lógica.

El segundo tipo de modificaciones eran medidas tendentes a resaltar lá macroestructura del texto y fueron: utilización de la repetición, la redundancia y el subrayado; y utilización de ideas-clave en el margen izquierdo del texto. Por último, el tercer tipo de modificaciones que ambos textos experimentales recibieron consistió en la simplificación léxica y sintáctica: utilizamos proposiciones más cortas y sencillas, eliminamos complejidades sintácticas sin afectar al contenido del texto, eliminamos algunos vocablos sustituyéndolos por términos más habituales manteniendo el contenido semántico.

Además de estas modificaciones utilizadas en ambos textos experimentales, el texto $B$ va precedido de un esquema y el texto $C$ incluye objetivos intercalados.

El diseño experimental fue de tres grupos (control A, experimental B y experimental C) con medidas repetidas (dos ocasiones de medida: postest y demora de una semana). Para la medida de la variable dependiente se utilizó tanto en el postest como en la demora, una prueba de recuerdo libre, y otra de reconocimiento. Para esta última se elaboró una prueba objetiva de 35 ítems y 3 alternativas. Se controló la covariable inteligencia verbal mediante las pruebas de palabra diferente y vocabulario del test TEA-3.

Además, para comprobar la eficacia diferencial de los textos utilizamos dos tipos de puntuaciones: las puntuaciones en las proposiciones cubiertas por el esquema más las proposiciones cubiertas por los objetivos $(\mathrm{E}+\mathrm{O})$; y las proposiciones cubiertas por ambos, el esquema y los objetivos ( $\mathrm{E}$ y $\mathrm{O}$ ). Las proposiciones cubiertas por el esquema más los objetivos $(\mathrm{E}+\mathrm{O})$ son todas las proposiciones destacadas por algunos de los tratamientos experimentales, incluyendo, las proposiciones destacadas por el tratamiento experimental, común a ambos grupos ( $\mathrm{B}$ y $\mathrm{C}$ ); es decir, aquellas proposiciones de la macroestructura que fueron resaltadas en el tratamiento experimental común mediante repetición, redundancia, subrayado y utilización de ideas-clave, y que fueron también resaltadas en el esquema, en los objetivos o en ambos. Las proposiciones cubiertas por ambos, el esquema y los objetivos ( $\mathrm{E}$ y $\mathrm{O}$ ) permiten una comparación en las mismas proposiciones de la eficacia de ambas técnicas ${ }^{1}$.

\section{Sujetos y procedimiento}

Los sujetos fueron alumnos de la UNED del Centro Asociado de Madrid. El número de sujetos que participaron en la primera sesión fue de 57, quedando asignados aleatoriamente del siguiente modo: grupo A, 19 sujetos (Edad media: 35,1; desviación típica, 8,6); grupo B, 19 sujetos (Edad media: 35,3; desviación típica, 10,1); grupo $C, 19$ sujetos (Edad media: 30,5; desviación típica:9). Hubo una mortalidad experimental de 12 sujetos, quedando los grupos en la segunda sesión (demora de una semana) como sigue: grupo A, 14 sujetos; grupo $\mathrm{B}, 15$ sujetos; grupo $\mathrm{C}, 16$ sujetos.

En la presentación del experimento se resaltaba la importancia de la investigación para la mejora del material didáctico de la UNED, se hacía una breve 
descripción de la secuencia de tareas y se insistía en la necesidad de participar en la segunda sesión experimental una semana más tarde. A continuación se entregaba a los sujetos una hoja de respuestas y realizaban el test de inteligencia verbal. Posteriormente se les entregaba el texto, asignándoles aleatoriamente a cada uno de los grupos ( $\mathrm{A}, \mathrm{B}$ o $\mathrm{C}$ ) dándoles doce minutos para su lectura. Se leían en voz alta las instrucciones y se aclaraban las dudas existentes. Se resaltaba el que los sujetos deberían lograr extraer la máxima información posible de los textos haciendo una lectura comprensiva. Tras un breve descanso se les pasaba la prueba de recuerdo libre, y finalmente, la de reconocimiento, otorgándoseles un máximo de 20 minutos para cada una. En la prueba de recuerdo libre se les pedía que reflejaran lo más exactamente posible el contenido del texto que habían leído. Al finalizar la sesión se les recordaba que una semana más tarde deberían de volver, aunque no se les decía cuál iba a ser su tarea. En la segunda sesión experimental se les volvían a pasar las pruebas de recuerdo libre y de reconocimiento.

\section{Análisis de los resultados}

Las tablas I y II muestran los resultados globales de los distintos grupos. En la prueba de reconocimiento en el postest, el grupo B fue el que mayor puntuación obtuvo, seguido del $\mathrm{C}$ y del A; en la demora, las puntuaciones del grupo A y $\mathrm{B}$ son muy semejantes y algo menores las del grupo C. En cuanto a la prueba de recuerdo libre, en el postest las puntuaciones obtenidas por el grupo B son superiores a las de los grupos A y C; en la demora se mantiene esta misma pauta de resultados pero algo disminuidos. En las pruebas de inteligencia verbal, el grupo $\mathrm{C}$ consiguió una puntuación más alta, seguido del $\mathrm{B}$ y del $\mathrm{A}$.

En la tabla II se pueden ver las correlaciones en cada uno de los grupos entre las puntuaciones de las medidas de la variable dependiente y las puntuaciones de la inteligencia verbal. El grupo A obtuvo las correlaciones más altas, siendo todas significativas $(\mathrm{p}<0,005)$. En el grupo $\mathrm{C}$ las correlaciones son más bajas, no alcanzando la significación en el postest. Por último, en el grupo B las correlaciones son cercanas a cero y en ningún caso significativas. Esta pauta diferencial de correlaciones será motivo de comentario posterior por su coherencia con nuestras hipótesis.

TABLA I

Resultados totales y medias en las pruebas de reconocimiento, recuerdo libre y factor verbal del TEA-3

\begin{tabular}{lcccccc}
\hline & & \multicolumn{2}{c}{ Reconocimiento } & \multicolumn{2}{c}{ Recuerdo libre } \\
Postest & Demora & Postest & Demora** & $\begin{array}{c}\text { Factor } \\
\text { verbal } \\
\text { TEA-3 }\end{array}$ \\
\hline Grupo A & Total & 447 & 331 & 203,5 & 109,5 & \\
& $\mathrm{M}$ & 23,52 & 23,64 & 10,71 & 7,82 & 121,64 \\
Grupo B & Total & 479 & 373 & 251 & 133,5 & \\
& $\mathrm{M}$ & 25,21 & 23,31 & 13,21 & 8,90 & 126,13 \\
Grupo C & Total & 468 & 353 & 205 & 105 & \\
& $\mathrm{M}$ & 24,68 & 22,06 & 10,78 & 6,76 & 127,17 \\
\hline
\end{tabular}

* Grupo A N = 14; Grupo B N = 16; Grupo C N = 16 .

** Grupo A N $=14$; Grupo B N = 15; Grupo $C N=15$. 


\section{TABLA II}

Correlaciones entre las pruebas de recuerdo y el CIV; correlaciones entre las pruebas de recuerdo

\begin{tabular}{|c|c|c|c|c|c|c|c|}
\hline \multirow[b]{3}{*}{ Grupo A } & \multicolumn{2}{|c|}{ Reconocimiento } & \multicolumn{2}{|c|}{ Recuerdo libre } & & & \\
\hline & & & & & & & \\
\hline & $0,668 * \star \star \star$ & $0,647 * * *$ & $0,645 * * \star$ & $0,614 * * *$ & & Postest & Demora \\
\hline Grupo B & 0,006 & 0,1 & 0,056 & 0,158 & $\begin{array}{l}\text { Recon./ } \\
\text { R. lib. } * \star *\end{array}$ & 0,734 & 0,592 \\
\hline Grupo C & 0,384 * & $0,495 * *$ & 0,223 & 0,419 * & & & \\
\hline
\end{tabular}

\section{FIGURA 1}

\section{DE RECUERDO CUANTITATIVO}

PUNTUUACION

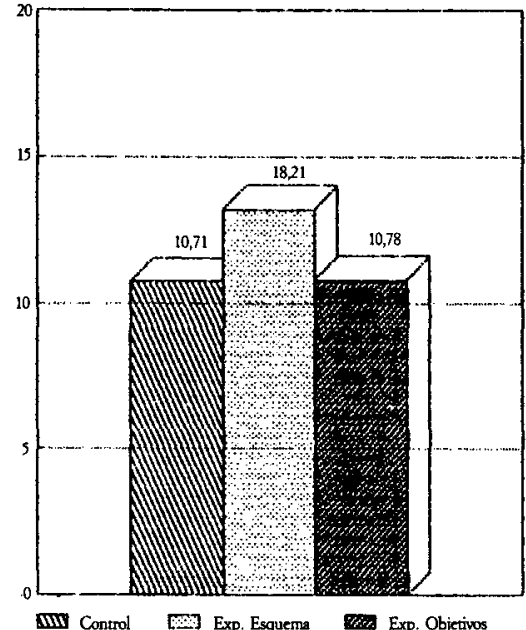

BLOQUES

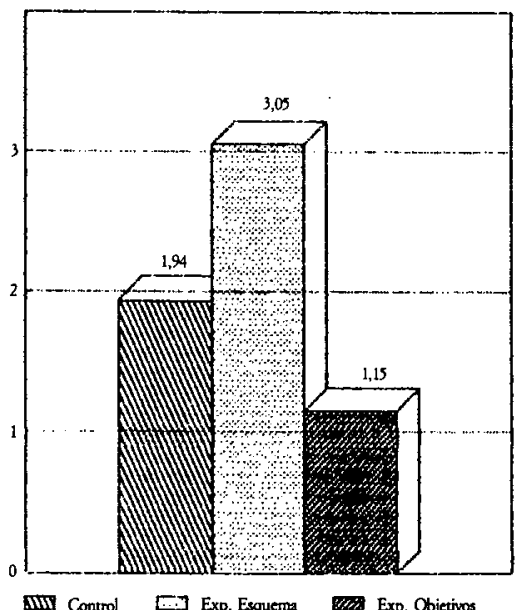

Puntuaciones medias de los grupos en la prueba de recuerdo libre en el postest.
ESCENARIOS

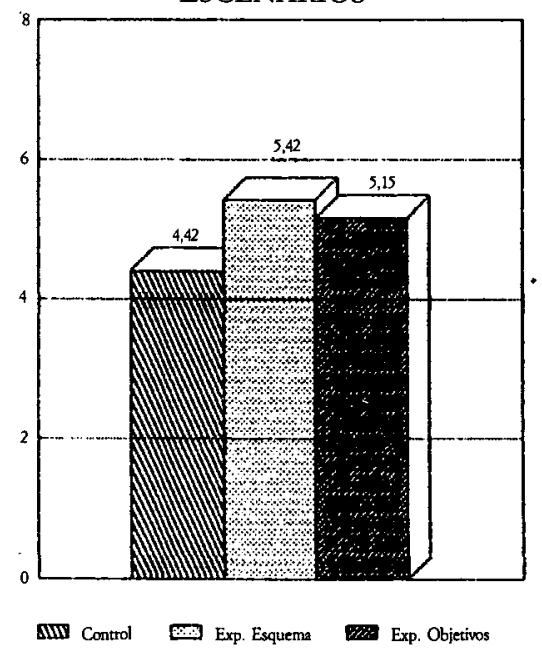

INFERENCIAS

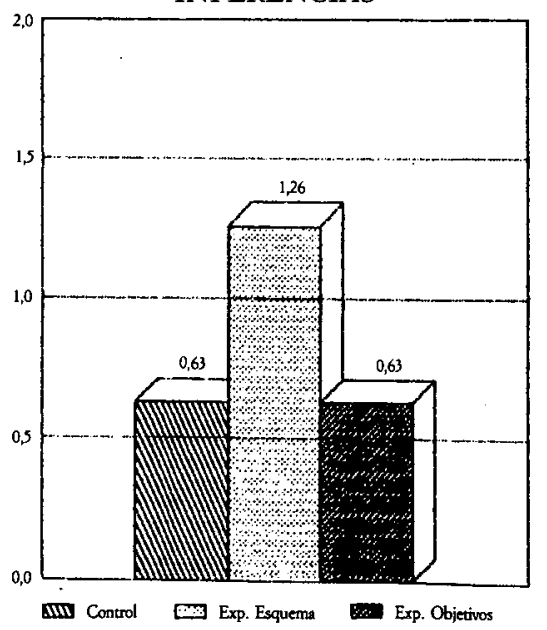


En cuanto a las correlaciones entre la prueba de reconocimiento y la de recuerdo libre fueron ambas positivas y de alto nivel de significación $(p<0,005)$ tanto en el postest como en la demora.

Se realizaron análisis de varianza tanto en la prueba de reconocimiento como en la de recuerdo libre $y$, aunque ninguno de ellos resultó significativo, la pauta de resultados es consistentemente favorable al grupo B.

Como se había explicado el cómputo de los resultados contenía diferentes niveles, además del recuerdo cuantitativo o puntuación total, obtuvimos una puntuación en escenarios, bloques e inferencias. En la figura 1 pueden observarse los resultados en el postest de la prueba de recuerdo libre que mantienen una pauta favorable al grupo experimental $\mathrm{B}$ en los distintos niveles de análisis del texto. Para comprobar la significación de los resultados se realizaron dos ANOVAS: uno con la suma de las tres puntuaciones (escenarios + bloques + inferencias) que puede ser considerada como una medida de memoria estructural y comprensión; en el segundo, tan sólo se consideraron las inferencias, con lo que podríamos obtener un índice de comprensión. En el primer caso el análisis de varianza resultó significativo $(F=2,55 ; \mathrm{p}<0,1)$; el contraste de las medias no obtuvo significación. El segundo análisis de varianza resultó significativo ( $\mathrm{F}=$ 3,55; $\mathrm{p}<0,005$ ), así como el contraste de medias (Prueba de Scheffé: $F b=2,33$; $\mathrm{Fb}-=2,33 ; \mathrm{p}<0,1)$. En la demora los resultados siguen la misma pauta pero disminuidos; los análisis de varianza realizados no fueron significativos.

En cuanto a los resultados del aprendizaje intencional, es decir, aquellas proposiciones afectadas directamente por el tratamiento experimental, realizamos los dos tipos de análisis que describimos anteriormente para comprobar la eficacia diferencial de las ayudas.

La tabla III contiene el resumen de la significación de los distintos contrastes de medias en el aprendizaje intencional y el aprendizaje incidental.

Los resultados de las proposiciones cubiertas por el esquema más los objetivos $(E+O)$, tanto en el postest como en la demora, son favorables al grupo $\mathrm{B}$, seguido del $\mathrm{C}$ y por último del $\mathrm{A}$. Resultaron significativas las diferencias B-A $(p<0,05)$ en el postest y B - C $(p<0,1)$ en la demora. Los resultados de aprendizaje incidental muestran una pauta desfavorable al grupo $\mathrm{C}$ ( $\mathrm{A}$ - $\mathrm{C}$, postest: $\mathrm{p}<0,1$; A - C, demora: $p<0,025$; B - C, postest: $\mathrm{p}<0,05)$ y con diferencias apenas inexistentes entre A y $B$. Estos resultados muestran que el tratamiento experimental ha producido en el grupo B una mejora en el aprendizaje intencional sin disminuir el aprendizaje incidental, al menos en el postest.

TABLA III

Significación de los contrastes entre medias. Aprendizaje intencional e incidental

\begin{tabular}{llllll}
\hline & & & B - A & C - A & B - C \\
\hline Aprendizaje & E + O & $\begin{array}{l}\text { Postest } \\
\text { Demora }\end{array}$ & $\begin{array}{l}<0,05 \\
<0,15\end{array}$ & & $<0,1$ \\
& E y O & $\begin{array}{l}\text { Postest } \\
\text { Demora }\end{array}$ & $<0,1$ & $<0,15$ & $<0,1$ \\
\hline \multirow{2}{*}{$\begin{array}{l}\text { Aprendizaje } \\
\text { incidental }\end{array}$} & $\mathrm{E}+\mathrm{O}$ & $\begin{array}{l}\text { Postest } \\
\text { Demora }\end{array}$ & & $<0,1$ & $<0,05$ \\
& $\mathrm{E} y \mathrm{O}$ & $\begin{array}{l}\text { Postest } \\
\text { Demora }\end{array}$ & & $<0,025$ & $<0,15$ \\
\hline
\end{tabular}


Las proposiciones cubiertas por ambas técnicas, esquema y objetivos (E y $\mathrm{O}$ ), han permitido la comparación de su eficacia diferencial mostrando la superioridad del grupo B (esquema) sobre el C (objetivos). Destacan, de nuevo, las bajas puntuaciones en aprendizaje incidental del grupo $\mathrm{C}$ y la disminución del grupo $\mathrm{B}$ entre postest y demora. En aprendizaje intencional en el postest las puntuaciones más altas las ha obtenido el grupo $\mathrm{B}$ aunque con diferencias prácticamente inexistentes respecto del grupo $\mathrm{C}$. En la demora, las puntuaciones obtenidas por el grupo B son claramente más altas que las de los otros dos grupos. Las diferencias de medias $B$ - A fueron significativas en el postest y en la demora $(\mathrm{p}<0,1)$; la diferencia $B$ - $C$ fue significativa en la demora ( $p<0,1)$.

En cuanto al aprendizaje incidental las puntuaciones más altas en el postest son las del grupo B, seguido del A y por último, del C; alcanzando la significación la diferencia B - C $(p<0,025)$. En la demora el orden fue el siguiente: grupo $A$, grupo $B$ y grupo $C$; aunque las diferencias entre $A$ y $B$ son mínimas y alcanzan sólo la significación las diferencias A - C $(\mathrm{p}<0,05)$ y B - C $(\mathrm{p}<0,1)$.

\section{Discusión}

La primera de nuestras hipótesis postulaba una mejora, tanto en el postest como en la demora, de los grupos experimentales $(\mathrm{B} \mathrm{y} \mathrm{C}$ ) respecto a el grupo control (A). Los resultados no han confirmado esta hipótesis, no pudiéndose rechazar la hipótesis nula acerca de las diferencias entre los grupos en la prueba de reconocimiento y en la de recuerdo libre, en el postest y en la demora. Sin embargo, existen dos características de los resultados que matizan esta conclusión global.

En primer lugar, el grupo B presenta una pauta de resultados que, aún sin alcanzar la significación, es constantemente superior a los otros dos grupos. Así, el grupo B alcanza puntuaciones superiores a los otros dos grupos en el postest (reconocimiento y recuerdo libre) y en la demora (recuerdo libre). Esta pauta diferencial de resultados en el grupo $\mathrm{B}$ queda puesta de manifiesto también si comparamos las correlaciones existentes entre el $\mathrm{CI}$ verbal y las puntuaciones en las pruebas de reconocimiento y recuerdo libre (tabla II). En el grupo A, y en menor medida en el grupo $\mathrm{C}$, las correlaciones son significativas sugiriendo que una parte importante de la varianza podría quedar explicada por la inteligencia verbal de los sujetos. Por el contrario, en el grupo B la inexistencia de correlaciones parece mostrar que las puntuaciones de los sujetos en las pruebas de recuerdo libre y reconocimiento no se han visto afectadas por su $\mathrm{CI}$ verbal. Esta falta de correlación en el grupo $B$ es coherente con una explicación de la mejora en los resultados de este grupo debido al tratamiento experimental.

En segundo lugar, destacan los pobres resultados obtenidos por el grupo experimental $\mathrm{C}$ en el postest y los resuitados inferiores, incluso a los del grupo control A, en la demora. El análisis de las correlaciones de las diferentes puntuaciones con el CI verbal no nos ofrece, en este caso ningún nuevo argumento que ayude a explicar estos resultados.

La segunda hipótesis perfilaba más nuestras predicciones al suponer que el grupo experimental B debería obtener mejores puntuaciones globales y de aprendizaje intencional que los otros dos grupos. Como se ha visto, la pauta de resultados del grupo B parece confirmar esta hipótesis ya que, además, los resultados 
en las medidas de memoria estructural y comprensión resultan significativamente favorables. Los sujetos del grupo B parecen mostrar en sus protocolos de recuerdo libre del postest una mejor memoria estructural (escenarios + bloques + inferencias) y especialmente una mejor comprensión medida mediante las inferencias. Estos resultados confirman nuestras previsiones acerca de las funciones del esquema proporcionando la estructura del texto y ayudando a construir una representación global del mismo. La misma pauta de resultados, aunque atenuada, se mantiene en la demora.

En cuanto al aprendizaje intencional la pauta de resultados general parece confirmar las predicciones de la segunda de nuestras hipótesis. En el caso de las proposiciones cubiertas por el esquema más los objetivos $(\mathrm{E}+\mathrm{O})$ que como ya dijimos, incluye aquellas proposiciones destacadas por el tratamiento experimental común, los resultados muestran claramente la superioridad del grupo B respecto a los otros dos grupos; alcanzando la significación las diferencias con el grupo control A en el postest $(p<0,05)$ y con el otro grupo experimental $C$ en la demora $(p<0,1)$. Los resultados del aprendizaje experimental del grupo $B$ al no existir diferencias con respecto al grupo control A y sin embargo, sí haberlas con respecto al grupo experimental $\mathrm{C}$ en el postest. Dicho de otra manera, la mejora en el aprendizaje intencional del grupo B no supone una disminución en las proposiciones no cubiertas por el tratamiento; es decir, no se hace a costa de una pérdida en aprendizaje incidental. Hay que destacar el hecho de que las proposiciones $\mathrm{E}+\mathrm{O}$ coinciden con la macroestructura del texto de donde proviene la importancia de los resultados encontrados con el grupo B, en contraposición al grupo $\mathrm{C}$.

Nuestro análisis de las proposiciones cubiertas por el esquema y los objeti$\operatorname{vos}(\mathrm{E}$ y $\mathrm{O})$ nos permite comparar la eficacia de ambos tratamientos. Los resultados muestran en el postest una gran semejanza en ambos grupos experimentales, aunque sólo el grupo B es significativamente superior a los otros dos grupos. Los resultados de aprendizaje incidental muestran que el grupo B se mantiene en niveles similares, o incluso superiores, a los del grupo control $A$, tanto en el postest como en la demora; el grupo $C$ muestra, de nuevo, una pérdida de aprendizaje incidental siendo significativamente inferior al grupo $\mathrm{B}$ en el postest y los dos grupos (A y B) en la demora. La comparación es totalmente favorable, otra vez, al grupo experimental B en el que se ha utilizado la técnica del esquema.

\section{SEGUNDO ESTUDIO}

\section{Objetivos e hipótesis}

Como decíamos anteriormente el objetivo principal de este segundo estudio es el de replicar algunos de los resultados más destacados del primero. Una de las variables que pensamos podría haber afectado a los resultados del primer estudio era la gran variabilidad de edades y de conocimientos previos de la muestra de sujetos alumnos de la UNED.

Un segundo objetivo fue ampliar el diseño a cuatro grupos experimentales $(2 \times 2)$. Con este nuevo diseño tratamos de comprobar experimentalmente la posibilidad observada de que los pobres resultados del grupo $\mathrm{C}$ se debieran a una interferencia entre el tratamiento experimental común y los objetivos. 
Las hipótesis de este estudio varían poco con respecto a las del primer estudio. El nuevo diseño permitirá distinguir si las diferencias significativas que se hallen se deberán a las características del texto (texto control o texto con tratamiento experimental común) o al tipo de ayuda experimental (esquema u objetivos). La primera hipótesis sostiene una mayor eficacia del esquema con respecto a los objetivos. La segunda postula la eficacia del texto experimental frente al texto control.

\section{METODO}

\section{Materiales y diseño}

Los materiales no difirieron de los del primer estudio. El diseño experimental fue factorial $2 \times 2$, siendo el primer factor el tipo de ayuda y el segundo el tipo de texto; de esta manera obtuvimos cuatro grupos:

- grupo A; texto control más un esquema previo.

- grupo B: texto experimental más un esquema previo.

- grupo C: texto. experimental más objetivos intercalados en el texto

- grupo D: texto control más objetivos intercalados en el texto.

Dados los objetivos del estudio se prescindió de la demora y de la prueba de reconocimiento. Por tanto los sujetos sólo respondieron en esta ocasión a una prueba de recuerdo libre.

Se mantuvieron los dos tipos de medida diferentes con los que pretendemos controlar la eficacia diferencial de los tipos de ayuda: las cubiertas por el esquema más los objetivos $(\mathrm{E}+\mathrm{O})$ y las proposiciones cubiertas por el esquema y los objetivos (E y O).

\section{Sujetos y procedimiento}

Los sujetos eran alumnos de tercero de BUP de un instituto de clase media de Madrid. Participaron un total de 105 sujetos que fueron asignados aleatoriamente a las cuatro condiciones experimentales como sigue: grupo A, 27 sujetos; grupo $B, 26$ sujetos, grupo $C, 28$ sujetos y grupo D, 24 sujetos. La edad media fue de 16,9; no encontrándose diferencias entre los grupos. Esta muestra, si bien garantiza la homogeneidad producirá como veremos más adelante, algunos resultados que tienen bastante que ver con los conocimientos previos de los sujetos.

El procedimiento fue, con las simplificaciones introducidas, igual que en el primer estudio. Se leían las instrucciones en voz alta, se aclaraban las dudas, se les insistía en que realizaran una lectura comprensiva e intentaran retener la máxima información posible. $\mathrm{Al}$ responder a la prueba de recuerdo libre, se les pedía, del mismo modo, que reflejasen lo más fielmente el texto que habían leído, en un tiempo de veinte minutos. 


\section{Análisis de los resultados}

En la figura 2 pueden observarse los resultados en puntuación total, escenarios, bloques y «mestre»; esta última, consiste, en este segundo estudio, en una medida que combina la puntuación total, los escenarios y los bloques y pretende medir la memoria estructural y la comprensión de los sujetos. La pauta de resultados es favorable a los grupos del esquema ( $\mathrm{A} \mathrm{y} \mathrm{B}$ ), confirmando nuestra primera hipótesis y coincidiendo de forma general con los resultados del primer estudio. Del mismo modo, respecto a nuestra segunda hipótesis, los resultados confirman la mayor eficacia de los dos textos experimentales frente a los dos de control.

\section{FigURA 2}

\section{PUNTUACION \\ DE RECUERDO CUANTITATIVO}

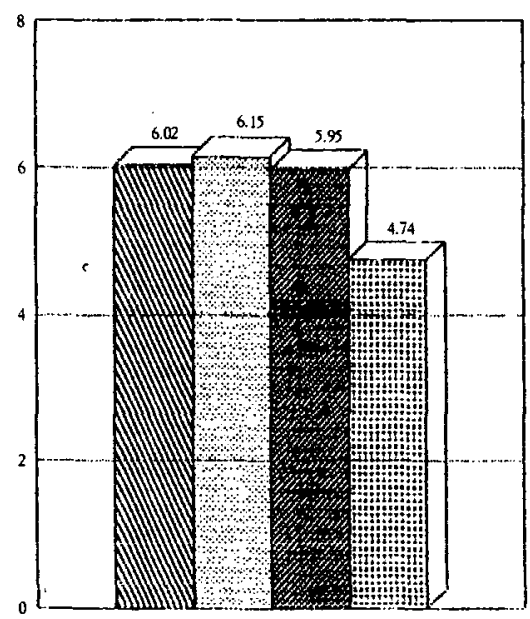

MIN O.Esq Ex.Esq Ex.Obj E. Ebj

\section{BLOQUES}

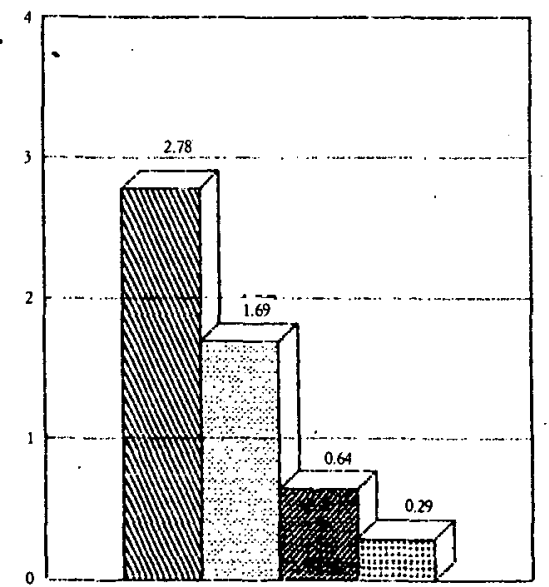

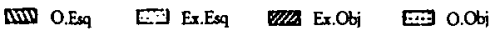

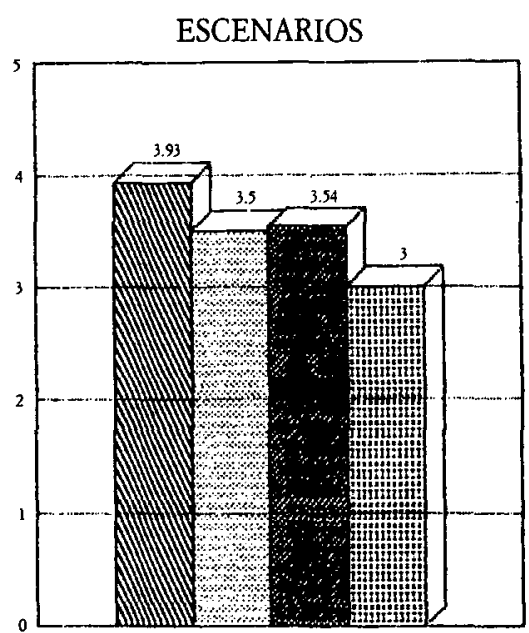

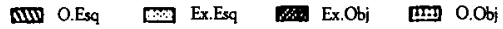

MESTRE

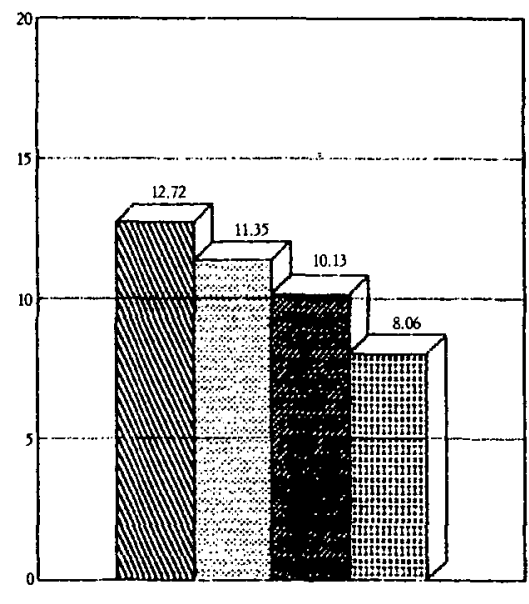

IIII O.Esq 0 Ex.Esq Ex.Obj $0.06 j$ 
El ANOVA $(2 \times 2)$ realizado con la puntuación total de recuerdo cuantitativo no fue significativo ( $F=1,434 ; p=0,237$ ), si bien los resultados de los grupos del esquema son superiores a los de objetivos, dándose el orden $\mathrm{B}, \mathrm{A}, \mathrm{C}$ y D.

Respecto de los escenarios, el ANOVA $(2 \times 2)$, realizado tampoco fue significativo $(F=0,755 ; p=0,522)$; el orden de los grupos fue, de nuevo, favorable a los grupos del esquema (A, B, C y D).

Los bloques produjeron una distribución no homocedástica por lo que utilizamos una prueba no paramétrica para contrastar los dos factores. La prueba de Chi-cuadrado sólo resultó significativa en el factor ayuda (chi-cuadrado $=$ 28.38026, G.L. $=5, \mathrm{p}<0,00001)$ mientras que la influencia del factor texto no obtuvo la significación. El orden en las puntuaciones (A, B, C y D) favoreció de nuevo a los grupos del esquema y todos los contrastes entre los grupos, mediante la prueba $U$ de Mann-Whitney fueron altamente significativos excepto la diferencia entre el grupo $\mathrm{C}$ y el grupo D.

La puntuación «mestre» produjo, del mismo modo, diferencias significativas (ANOVA $2 \times 2$; efecto principal $\mathrm{F}=8,588, \mathrm{p}<0,0001$ ); el factor ayuda resultó significativo $(\mathrm{F}=16.527, \mathrm{p}<0,0001)$ mientras que la condición de texto (control o tratamiento experimental común) no influyó significativamente en los resultados. El orden fue igual a la anterior puntuación y en este caso tan sólo la diferencia entre los grupos A y D resultó ser significativa $(\mathrm{p}<0,05)$.

Si en el primer estudio pensamos que la variabilidad de los sujetos que componían la muestra podía haber determinado algunos resultados, conseguir homogeneidad, supuso, en este caso, que el nivel de conocimientos previos de los sujetos afectase a los resultados. El número medio de proposiciones que recordaban los sujetos era casi la mitad que en el primer estudio. Por otra parte, las correlaciones entre el número de proposiciones recordadas y las distintas puntuaciones de aprendizaje intencional e incidental resultaron altas y significativas (con esquema intencional, 0,71; esquema incidental, 0,79; objetivo intencional, 0,61 ; objetivo incidental, 0,82 ; esquemas más objetivos intencional, 0,70 ; esquemas más objetivos incidental, 0,$79 ; \mathrm{p}<0,001$ para todas las correlaciones) por lo que nos pareció aconsejable realizar dos tipos de análisis estadístico. La variante consistía en introducir como control estadístico la covariable número de proposiciones recordadas. Los resultados mostraron que en el análisis con la covariable las tendencias se confirmaban, obteniéndose diferencias significativas.

En cuanto al aprendizaje intencional e incidental se efectuaron los mismos tipos de medida que en el primer estudio. Los resultados que se detallan a continuación tienen en cuenta la covariable número de proposiciones. En las proposiciones cubiertas por cualquiera de los tratamientos experimentales $(E+O$; esquemas, objetivos o tratamiento experimental común) los efectos principales rozaron la significación (ANCOVA; $F=3,031 ; p=0,053$ ) siendo el factor texto significativo (ANCOVA; $F=5,926 ; p=0,017$ ). Ni la ayuda ni la interacción entre ambos factores resultó significativa. Parece ser, que el hecho de que el tratamiento experimental común coincida con el esquema o los objetivos es decir, que mediante los tres se destaque la macroestructura del texto de forma a veces redundante, ha podido afectar al contraste del factor ayuda y de la interacción. En este caso, como en posteriores, el efecto suelo (en cuanto al número de proposiciones recordadas) no ha permitido un contraste correcto entre los diferentes tipos de ayuda y sí un contraste más grueso; del que se benefician mayor número de proposiciones, como es el concerniente al tratamiento experimental común. 
Las proposiciones cubiertas por ambos tratamientos, esquema y objeti$\operatorname{vos}(\mathrm{E}$ y $\mathrm{O}$ ), permiten una comparación en las mismas proposiciones de la eficacia de estas técnicas. Si las diferencias del efecto principal resultan significativas ( $F=4,149, p=0,019)$ lo son, sin embargo, por el fuerte contraste en el factor texto $(F=8,193, p=0,005)$; es decir, no aparecen apenas proposiciones que nos permitan una comparación del factor ayuda sin que éstas coincidan con las del tratamiento experimental común. Respecto al aprendizaje incidental ninguno de los contrastes resulta significativo.

\section{Discusión}

En líneas generales, desde una perspectiva de replicación experimental podemos afirmar que, si bien los resultados no alcanzan siempre los niveles de significación sí son consistentes con los del primer estudio, confirmando nuestras dos hipótesis.

Como adelantábamos anteriormente, el bajo nivel de conocimientos previos de los sujetos parece haber restado amplitud a las diferencias entre los distintos grupos. En este sentido debe observarse que si en este segundo estudio no ha aparecido el análisis de las inferencias, se debe, sencillamente, a que los sujetos no han podido producirlas dado sus escasos conocimientos sobre el tema; recuérdese que el ANOVA de la producción de tales inferencias nos permitió obtener un índice de comprensión en el primer estudio. Otro resultado relacionado con esta línea de argumentación puede obtenerse al observar que sólo en el caso de la más alta de las medidas de cómputo proposicional (bloques), se hayan producido diferencias significativas. Estas diferencias además, han resultado significativas entre todos los grupos menos la resultante de lós dos grupos de objetivos. La memoria de la macroestructura del texto ha sido perfectamente cubierta en los grupos del esquema. Sin embargo, a pesar de esto, el esquema no ha podido activar los conocimientos previos donde los nuevos conceptos podrían haber sido asimilados con mayor abundancia de detalles, en este caso, mayor número de proposiciones. Parece ser que el nivel de conocimientos de los sujetos respecto a este tema era demasiado pobre como para que la interacción entre previos y nuevos conceptos pudiera ser más rica.

El efecto suelo parece también haber mediado en los contrastes que sobre el aprendizaje intencional e incidental hemos llevado a cabo. Al igual que en el primer estudio los resultados son consistentemente favorables a los grupos del esquema, aunque sólo los contrastes gruesos (texto control o experimental) hayan obtenido la significación. Por otra parte, la utilización de un diseño factorial ha permitido distinguir con más claridad alguno de los resultados. En el aprendizaje intencional los grupos con tratamiento experimental común ( $\mathrm{B}$ y $\mathrm{C}$ ) han obtenido siempre los mejores resultados lo que confirma plenamente nuestra segunda hipótesis. El grupo experimental con esquema resulta superior en ambas medidas de aprendizaje intencional frente al grupo experimental con objetivos; siendo el grupo control-esquema siempre superior al control-objetivos. En el aprendizaje incidental, si bien no se dan diferencias significativas, los resultados favorecen claramente a los grupos del esquema. Por tanto la pauta de resultados coincide con la del primer estudio y nuestra hipótesis sobre la mayor eficacia del esquema se ve de nuevo reforzada. 


\section{CONCLUSIONES GENERALES}

Nos propusimos como objetivo evaluar las diferencias de dos tipos de ayuda -esquemas y objetivos - en su eficacia para optimizar los procesos de almacenamiento y recuperación de textos expositivos. Un comentario previo a cualquier conclusión de corte general debe destacar la gran complejidad y matices que se dan cita en este tipo de tareas.

De los resultados expuestos puede desprenderse que, aunque no siempre significativa, la pauta de resultados que producen los dos estudios es consistente y favorable al esquema. Nuestra explicación sobre cómo ha funcionado el esquema en sus distintas condiciones es la que a continuación detallamos. El esquema debía de proveer al sujeto de la estructura del texto y ayudarlo a centrarse en sus aspectos más relevantes, es decir, la macroestructura del texto. Efectivamente, el esquema parece haber actuado sobre la memoria estructural y la comprensión principalmente y sin obtener la significación a través de éstas, en el recuerdo cuantitativo. Esta interpretación de los datos se ve reforzada por el resultado que los grupos del esquema han obtenido en el aprendizaje incidental, que muestran que no hay una pérdida del mismo; de lo que deducimos que la actuación del esquema es de corte general y no sólo específica. Además, en el primer estudio, donde introdujimos la medida demorada, la pérdida del grupo B (experimental-esquema) es mínima, resultado que contrasta con las pérdidas que sufrió el grupo C (experimental-objetivos).

La falta de eficacia de los objetivos parece otro tema necesitado de una explicación. Una posible explicación podría ser que nuestros objetivos hayan sido demasiado densos y generales, perdiendo, por tanto, su eficacia diferencial en aprendizaje intencional y sin, por ser más generales, lograr una mejora de la comprensión y el recuerdo global. Otra posible explicación podría provenir de una interferencia del tratamiento experimental común y los objetivos. Existen estudios que ofrecen resultados que hacen plausible esta interpretación (Brooks y cols., 1983); sin embargo, en nuestro segundo estudio, a pesar de las limitaciones ya apuntadas, puede observarse que los grupos de los objetivos mantienen las puntuaciones más bajas. El grupo control-objetivos (D) obtiene en todas las medidas la puntuación más baja, siendo siempre el último de los grupos. Por otra parte, en el primer estudio se observa una disminución en los resultados de la demora que pueden ser explicados en términos de dificultades en la recuperación.

De este modo, dos pueden ser las conclusiones generales. En primer lugar, resaltar la eficacia del esquema frente a la de los objetivos, especialmente en la comprensión y recuerdo global y la memoria de la macroestructura del texto. La segunda de las conclusiones hace referencia a la forma en que ha actuado cada una de las ayudas. El esquema parece fomentar las estrategias cognoscitivas de corte deductivo, activando, al menos en el primer estudio, las estructuras previas de conocimientos necesarias para el procesamiento del texto. En ambos estudios puede observarse la capacidad del esquema para transmitir la macroestructura del texto. En el caso de los grupos que tenían el tratamiento de los objetivos, la actuación de los sujetos parece indicar que la densidad de los objetivos utilizados no ha permitido la sustancial mejora que se esperaba en el aprendizaje intencional, produciéndose un procesamiento de tipo puntual, y fragmentario que no favoreció la comprensión, ni la memoria, global del texto.

En resumen, nuestra conclusión general, sujeta a posteriores estudios que 
permitan una mayor precisión, postula que el esquema actúa favoreciendo el normal procesamiento del texto por parte de los sujetos, en forma coherente con nuestro conocimiento sobre cómo se realiza la comprensión de textos; mientras que los objetivos poseen una utilidad limitada ya que no están basados en una adecuada teoría sobre el procesamiento y comprensión de textos (Resnick, 1984).

\section{Notas}

' Una descripción más detallada de este primer estudio, el contexto teórico global y la lista de las proposiciones del texto, así como los propios textos utilizados puede encontrarse en García Madruga y Martín Cordero, 1987.

\section{Referencias}

Ausubel, D. P. (1968). Educational Psychology: A cognitive view. Nueva York, Holt, Rinehart y Winston.

Ausubel, D. P., Novak, J. D. y Hanesian, H. (1978). Educational Psychology: A cognitive view, Nueva York, Holt, Rinehart and Winston.

BRANSFORD, J. D., BARCLAY, B. D. y FRANKS, J. J. (1972). «Sentence memory: a constructive versus interpretative approach", Cognitive Psychology, 3, 193-209.

BRANSFORD, J. D. y JOHNSON, M. K. (1973). «Considerations of some problems in comprehension.» En W. B. Chase (ed.), Visual information processing, Nueva York, Academic Press, 1973.

- (1972). «Contextual prerequisites for understanding: Some investigations of comprehension and recalls. Journal of Verbal Learning and Verbal Bebavior, 11, 717-726.

BRANSFORD, J. D. y MCARRELL, N. (1975). "A sketch of a cognitive approach to comprehension: Some thoughts about understanding what it means to comprehend". En W. B. Weimer y D. S. Palermo (eds.), Cognition and the Symbolic Processes. Hillsdale, Nueva York: LEA, 1975.

BROOKS, L. W. y cols. (1983). «Effects of headings on text processing». Journal of Educational Psycho$\log y, 75,2,292-302$.

CIRILO, R. H. y Foss, D. J. (1980). «Text structures and reading time for sentences». Journal of Verbal Learning and Verbal Behavior, 19, 96-109

Clark, H. H. (1975). «Bridging». En P. N. Johnson-Laird y P. C. Wason (eds.), Thinking. Readings in cognitive science, Cambridge: Cambridge University Press, 1977.

GAGNE, R. M. (1977). The conditions of Learning. Nueva York: Holt. Rinehart y Winston.

Garcia Madruga, J. A. y Martin Cordero (1987). Aprendizaje, Comprensión y Retención de texios, Madrid, UNED.

JOHNSON-LAIRD, P. N. (1983). Mental models. Towards a cognitive science on language. Inference and consciousness. Cambridge, Cambridge University Press.

JUST, M. A. y CARPENTER, P. A. (1987). The psichology of reading and language comprebension, Newton, Mass.: Allyn y Bacon.

KINTSCH, W. y VAN DIJK, T. A. (1978). «Toward a model of text comprehension and production». Psycbological Review, 85, 363-394.

MEYER, B. J. F. (1984). «Text dimensions and cognitive processing». En: H. Mandl, N. L. Stein y T. Trabasso (eds.). Learning and Comprebension of Text, Hillsdale, Nueva York, LEA, 1984.

MinSKY, M. (1975). «A framework for representing knowledge.» En: P. A. Winston (ed.), The psychology of computer vision. Nueva York: McGraw-Hill, 1975.

NovaK, J. D. (1977). A theory of education. Cornell: Cornell University Press.

RESNICK, L. B. (1984). «Comprehending and learning: implications for a cognitive theory of instruction." En H. Mandl, N. L. Stein y T. Trabasso (eds.): Learning and comprehension of text. Hillsdale, N. J.: LEA.

ROTHKOPE, E. Z. (1970). "The conceptos of mathemagenic activities». Review of educational research, 40, 325-336.

ROTHKOPF, E. Z. y KAPLAN, R. (1972). «Exploration of the effects of density and specificity of instructional objective on learning from text». Journal of educational psychology, 63, 4, 295-302.

RumelHART, D. E. (1975). «Notes on a schema for stories». En: D. G. Bobrow y A. Collins (eds.), Representing and Understanding: Studies in Cognitive Science, Nueva York, Academic Press, 1975.

RumelHART, D. E. (1980). «Schemata. The buildings blocks of cognition». En: R. J. Spiro (comp.), Theoretical issues in reading comprehension, New Jersey, LEA, 1980.

RUMELHART, D. E. y ORTONY, A. (1977): «The representation of knowledge in memory». En: R. 
C. Anderson, R. J. Spiro y W. E. Montague (eds.). Schooling and the acquisition of knouledge. Hillsdale, Nueva York, LEA.

SCHANK, R. C. y ABELSON, R. P. (1977). Scripts, Plans, Goals and Understanding: an enquiry into buman knouledge structure. Hillsdale, Nueva York: LEA.

SCHNOTZ, W. (1984). «Comparative instructional text organization». En : H. Mandl, N. L. Stein y T. Trabasso (eds.), Learning and comprebension of text. Hillsdale, Nueva York, LEA.

THORNDYKE, P. W. y HAYES.ROTH, B. (1979). "The use of schemata in the acquisition and transfer of knowledge». Cognitive Psychology, 11, 82-106.

VAN Dijk, T. A., (1983). «Epilogo a la edición castellana». En: T. A.Van Dijk, La ciencia del texto, Barcelona, Paidós.

VAN DiJK, T. A. y KinTSCH, W. (1983). Strategies of Discourse Comprebension, Nueva York, Academic Press.

\section{Extended summary}

This paper begins whith a brief theoretical review of the literature on text comprehension and memory. In this review we comment on the type of representation used for text information storage and on the important role that constructive processes have in this domain. Two trends of work converge on this issue: the increasing amount of theories and data on reading and text memory processes developed by cognitive psychology, and the work of educational psychologists interested in how to improve student's comprehension and retention of expository texts. Broadly speaking, the main agreement is that comprehension processes should be considered as the core problem in learning from text. In this sense, it is, the integration of new information in what is already know as the main target, that the first reading of a text should achieve: the second important point is that information in texts should be organized in such a way as to enable the reader to build a storage structure that will facilitate its retrieval in due time.

After this brief presentation of the theorethical context of our research, we will comment on the results of two experiments. These were developed the assess the influence on the comprehension and recall of expository prose of two kinds of manipulations performed to increase learning from texts. The first of these is lexical and sintactical simplification and the reordering of information in the text. The second intervention technique we have used is the introduction of different learning aids. In this respect, we have concentrated on the effects of learning objectives versus outlines.

Our first experiment was a three groups design in which we used two texts containing the same information though arranged in different form. The control text was a fragment from the developmental Psychology Units being used in our department. This text was sintactically and lexically simplified and its information rearranged to be used as our experimental text. In this first experiment the two treatment groups received the experimental text. In one of them the text was preceedeb by and outline while in the other learning objectives were included in the text instead of the outline. Our third group was a control using the standard text from the Units. Subjects in this experiment were Open University students, which meant a wide quite range of ages and study skills.

The second experiment consisted on a four groups design which compared the cross effects of type of text (standard v. experimental) and type of learning aid (outline v. objectives). Subjects in this second experiment were secondary education students. Texts used in both experiments were parsed into proposi- 


\section{4}

tions (idea-units) to allow the cuantification of recall protocols. This parsing also allowed us to evaluate, the recall of the original text organization in the form of its hierarchical structure. In one of the experiments, we also cuantified subject's intrusions in the recall protocols. The model of cuantification for the dependent variable was rather complicated, but it generated a wide variety of data. The main results from both experiments point in the same direction:

a) A good text organization (hierarchical) can save reader's cognitive resources which may then be used for a deeper comprehension of the text. That is, a good text (from a didactical standpoint) is one that allows the reader to use a mininum of his/her cognitive capacity in text decoding, or surface processing, and a maximum of cognitive capacity in text comprehension, or in linking text content to previously known information.

b) A related conclusion is that learning aids which contribute to a richer construction of a text model are of better use that those aids which aim at a more underlining of the important points in a text. That is to say, our results suggest that text outlines, or similar devices pointing to text macroestructure, produce better performance than do learning objectives. 